\title{
What's for dinner? Diet and potential trophic impact of an invasive anuran Hoplobatrachus tigerinus on the Andaman archipelago
}

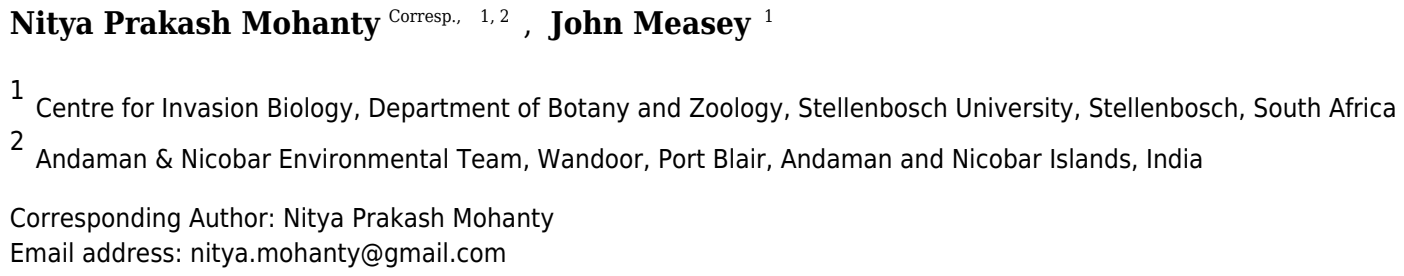

Amphibian invasions have considerable detrimental impacts on recipient ecosystems. However, reliable risk analysis of invasive amphibians still requires research on more nonnative amphibian species. An invasive population of the Indian bullfrog, Hoplobatrachus tigerinus, is currently spreading on the Andaman archipelago and may have significant trophic impacts on native anurans through competition and predation. We carried out diet analyses of the invasive $H$. tigerinus and native anurans, across four habitat types and two seasons; we hypothesized that i) small vertebrates constitute a majority of the $H$. tigerinus diet, particularly, by volume and ii) the diet of $H$. tigerinus significantly overlaps with the diet of native anurans, thereby, leading to potential competition. We assessed the diet of the invasive Hoplobatrachus tigerinus ( $n=358$ ), and individuals of the genera Limnonectes $(n=375)$ and Fejervarya $(n=65)$ and found a significant dietary overlap of $H$. tigerinus with only Limnonectes. Small vertebrates, including several endemic species, constituted the majority of $H$. tigerinus diet by volume, suggesting potential impact by predation. Prey consumption and electivity of the three anurans indicated a positive relationship between predator-prey body sizes. Individuals of Hoplobatrachus tigerinus and Fejervarya chose evasive prey, suggesting that these two taxa are mostly ambush predators; individuals of Limnonectes elected sedentary prey; although a large portion of its diet consisted of evasive prey, such electivity indicates 'active search' as its major foraging strategy. All three species of anurans mostly consumed terrestrial prey. This intensive study on a genus of newly invasive amphibian contributes to the knowledge on impacts of amphibian invasions, and elucidates the feeding ecology of $\mathrm{H}$. tigerinus, and species of the genera Limnonectes and Fejervarya. We also stress the necessity to evaluate prey availability and volume in future studies for meaningful insights into diet of amphibians. 
1 What's for dinner? - Diet and potential trophic impact of an invasive anuran Hoplobatrachus

2 tigerinus on the Andaman archipelago

3 Nitya Prakash Mohanty ${ }^{1,2}$, John Measey ${ }^{1}$

$4{ }^{1}$ Centre for Invasion Biology, Department of Botany \& Zoology, Stellenbosch University, South 5 Africa 7602.

$6{ }^{2}$ Andaman \& Nicobar Environment Team, Wandoor, South Andaman, Andaman and Nicobar 7 Islands 744103.

8 Corresponding Author: Nitya Prakash Mohanty, nitya.mohanty@gmail.com

9 (iD) $\underline{0000-0001-7768-6483}$

10 (iD) $\underline{0000-0001-9939-7615}$

11 ABSTRACT

Amphibian invasions have considerable detrimental impacts on recipient ecosystems. However, reliable risk analysis of invasive amphibians still requires research on more non-native amphibian species. An invasive population of the Indian bullfrog, Hoplobatrachus tigerinus, is currently spreading on the Andaman archipelago and may have significant trophic impacts on native anurans through competition and predation. We carried out diet analyses of the invasive H. tigerinus and native anurans, across four habitat types and two seasons; we hypothesized that i) small vertebrates constitute a majority of the $H$. tigerinus diet, particularly, by volume and ii) the diet of $H$. tigerinus significantly overlaps with the diet of native anurans, thereby, leading to potential competition. We assessed the diet of the invasive Hoplobatrachus tigerinus 
21 ( $n=358)$, and individuals of the genera Limnonectes $(n=375)$ and Fejervarya $(n=65)$ and found

22 a significant dietary overlap of $H$. tigerinus with only Limnonectes. Small vertebrates, including

23 several endemic species, constituted the majority of $H$. tigerinus diet by volume, suggesting

24 potential impact by predation. Diets of the three species were mostly governed by the positive

25 relationship between predator-prey body sizes. Individuals of Hoplobatrachus tigerinus and

26 Fejervarya chose evasive prey, suggesting that these two taxa are mostly ambush predators;

27 individuals of Limnonectes elected sedentary prey; although a large portion of its diet consisted of evasive prey, such electivity indicates 'active search' as its major foraging strategy. All three species of anurans mostly consumed terrestrial prey. This intensive study on a genus of newly invasive amphibian contributes to the knowledge on impacts of amphibian invasions, and elucidates the feeding ecology of $H$. tigerinus, and species of the genera Limnonectes and Fejervarya. We also stress the necessity to evaluate prey availability and volume in future studies for meaningful insights into diet of amphibians.

\section{INTRODUCTION}

Accelerating rates of biological invasions (Seebens et al., 2017) and their consequent negative impacts (Simberloff et al., 2013) have led to increased efforts towards pre-invasion risk assessment and prioritization based on impacts (Blackburn et al., 2014). Amphibian invasions have considerable detrimental impacts on recipient ecosystems (Pitt et al., 2005; Kraus, 2015), the magnitude of impact being comparable to that of invasive freshwater fish and birds (Measey et al., 2016). Impact mechanisms of amphibian invaders remain relatively understudied (Crossland et al., 2008) and are varied. Impact via predation and competition 
42 (sensu Blackburn et al., 2014) has been documented on invertebrates (Greenlees et al., 2006;

43 Choi and Beard 2012; Shine 2010), fishes (Lafferty and Page 1997), amphibians (Kats \& Ferrer,

44 2003; Wu et al., 2005; Measey et al., 2015; Liu et al., 2015; but see Greenlees et al., 2007) and birds (Boland, 2004), though other taxa may also be affected (Beard \& Pitt, 2005). Amphibian invaders may carry diseases (e.g. Batrachochytrium dendrobatidis; Garner et al., 2006; Liu et al., 2013) and cause reproductive interference (D'Amore et al., 2009), apart from several other ecological impacts (see Kraus, 2015 and Measey et al., 2016 for detailed assessments).

However, reliable risk analysis of invasive amphibians still requires research on more non-native amphibian species, as the existing knowledge on impacts is mostly based on the cane toad Rhinella marina and the American bullfrog Lithobates catesbeianus (Measey et al., 2016). Comparisons of impact across taxonomic groups for management prioritization (Blackburn et al., 2014; Kumschick et al., 2015) may also be impeded by the relatively understudied category of amphibian invasions as compared to other vertebrate invasions (Pyšek et al., 2008). This knowledge gap is further compounded by geographic biases in invasion research, with limited coverage in Asia and Africa (Pyšek et al., 2008); developing countries also have relatively less invasion research (Nunez \& Pauchard 2010; Measey et al., 2016).

An invasive population of the Indian bullfrog, Hoplobatrachus tigerinus (Daudin, 1802), is currently spreading on the Andaman archipelago, Bay of Bengal (Mohanty \& Measey, in review). The bullfrog was most likely introduced in early 2000 s and its exponential expansion has occurred since 2009, resulting in invasive populations on six out of the eight human inhabited islands of the Andaman archipelago (Mohanty \& Measey, in review). 'Contaminants' 
63 of fish culture trade and intentional 'release' are likely to be the primary pathways of

64 introduction and post-introduction dispersal, facilitating introductions from the Indian

65 mainland and inter-island transfers (Mohanty \& Measey, in review). The bullfrog has its native

66 range on the Indian sub-continent encompassing low to moderate elevations in Nepal, Bhutan,

67 Myanmar, Bangladesh, India, Pakistan, and Afghanistan (Dutta, 1997). The bullfrog has

68 previously been introduced to Madagascar (Glaw \& Vences, 2007), and possibly to the Maldives

69 (Dutta, 1997) and Laccadive Islands (Gardiner 1906). This large bodied frog (up to $160 \mathrm{~mm}$ ) has

high reproductive potential (up to 5750 eggs per clutch, Oliveira et al., 2017) and is uncommon

or absent in forested and coastal regions but occurs as a human commensal in plantations and

agricultural fields (Daniels 2005). It is considered a dietary generalist, feeding on invertebrates

and even large anurans such as Duttaphrynus melanostictus (Padhye et al., 2008; Datta \&

Khaledin, 2017); however, quantitative diet assessment with adequate sample size across

habitats and seasons is lacking (but see Khatiwada et al., 2016 for diet of $H$. tigerinus in rice

fields of Nepal).

Hoplobatrachus tigerinus on the Andaman archipelago co-occurs with native anurans of the

genera Duttaphrynus, Fejervarya, Limnonectes, and Microhyla (NPM unpublished data;

Harikrishnan et al., 2010). Given the large size of $H$. tigerinus, it is likely to feed on

proportionately large prey, including amphibians and other vertebrates (Datta \& Khaledin,

2017; Measey et al., 2015). The high volume of prey consumed by $H$. tigerinus (Padhye et al.,

2008) may lead to direct competition with native anurans, especially under relatively high 
85

86

87

be a generalist forager on terrestrial invertebrates (Hirai \& Matsui, 2001), Limnonectes spp. are known to feed on vertebrates in addition to arthropods (Emerson, Greene \& Charnov 1994). This leads us to expect a high diet overlap of native frogs belonging to Fejervarya and Limnonectes, with the generalist $H$. tigerinus. In terms of size, $H$. tigerinus is much larger than native anurans of the Andaman archipelago (Fig. 1) and may impact the native anurans through both predation and competition.

Niche overlap, in combination with prey availability (electivity), can be used to assess trophic competition between species (e.g. Vogt et al., 2017). In addition to taxonomic evaluation and enumeration of the prey consumed, it is crucial to consider prey volume and frequency of prey occurrence to ascertain overall importance of a particular category of prey (Hirschfeld \& Rödel, 2011; Boelter et al., 2012; Choi and Beard 2012). Classification by functional type (hardness and motility of prey) is useful in understanding predator behaviour (Toft 1980; Vanhooydonck et al., 2007; Carne \& Measey 2013). Further, seasonality in prey availability may influence diet in amphibians (Hodgkison \& Hero 2003; de Oliveira \& Haddad, 2015), therefore, there is also a need to assess diet across seasons, to fully capture the range of prey. Another important driver of prey choice may be the positive relationship between predator-prey body sizes (Werner et al., 1995; Wu et al., 2005).

We aimed to assess the trophic impact of the invasive Hoplobatrachus tigerinus on the native anurans of the Andaman Islands through predation and potential competition. We carried out diet analyses of the invasive $H$. tigerinus and native anurans, across four habitat types and two seasons, to ascertain the nature and magnitude of trophic impact. We hypothesized that i) 
106

107

108

109

110

111

112

113

114

115

116

117

118

119

120

121

122

123

124

125

126

small vertebrates constitute a majority of the $H$. tigerinus diet, particularly, by volume and ii)

the diet of $H$. tigerinus significantly overlaps with the diet of native anurans, thereby, leading to potential competition. Additionally, we aimed to characterize the predation behaviour of these anurans in terms of electivity and predation strategy (ambush or active search).

\section{METHODS}

We carried out the study in the Andaman archipelago for six months, from February to July 2017. The Andaman archipelago comprises nearly 300 islands (ca. $6400 \mathrm{~km}^{2}$ ) is situated between $10^{\circ} 30^{\prime} \mathrm{N}$ to $13^{\circ} 40^{\prime} \mathrm{N}$ and $92^{\circ} 10^{\prime} \mathrm{E}$ to $93^{\circ} 10^{\prime} \mathrm{E}$ (Fig. 2), which are part of the Indo-Burma biodiversity hotspot (Myers et al., 2000) with a 40\% endemism level in herpetofauna (Harikrishnan et al., 2010). The tropical archipelago receives an annual rainfall of $3000 \mathrm{~mm}$ to $3500 \mathrm{~mm}$ (Andrews and Sankaran 2002); primary and secondary forests encompass nearly $87 \%$ of the entire archipelago (Forest Statistics 2013), whereas the remaining human modified areas comprise of settlements, agricultural fields, and plantations. Of the nine species of native amphibians recorded, five species (Ingerana charelsdarwinii, Blythophryne beryet, Microhyla chakrapani, Kaloula ghoshi and Fejervarya andamanensis) are endemic to the Andaman Islands (Das 1999; Harikrishnan et al., 2010; Chandramouli et al., 2016; Chandramouli et al., 2018), however, taxonomic uncertainties still persist (Chandramouli et al, 2015; Harikrishnan Surendran, Pers. Comm.). Post-metamorphic frogs of the range restricted I. charlesdarwinii, the semi-arboreal B. beryet, the arboreal Kaloula ghosii and the littoral F. cancrivora are unlikely to co-occur with H. tigerinus at present (Das 1999; Chandramouli 2016; Chandramouli et al., 2016). Thus, we constrained our choice for comparative species to those which were strictly 
127 syntopic. As the taxonomy of the Andaman amphibians remains in flux, we limited our

128 identifications to the genus level for species belonging to the genera Fejervarya and

129 Limnonectes, which are pending formal re-assessments (Chandramouli et al., 2015). Currently,

130 L. doriae, L. hascheanus, Fejervarya limnocharis, F. andamanensis, and F. cancrivora are

131 considered members of these two genera in the Andaman Islands (Harikrishnan et al., 2010;

132 Harikrishnan Surendran, Pers. Comm.). Hereafter, Fejervarya spp. and Limnonectes spp. are

133 referred to as Fejervarya and Limnonectes, respectively.

134 We conducted the study at two sites (Webi and Karmatang) on Middle Andaman Island and at

135 one site (Wandoor) on South Andaman Island (Fig. 2). We chose sites with moderately old

136 invasions of $H$. tigerinus (more than 3 years since establishment; Mohanty \& Measey in review),

137 assuming that a relatively longer time since establishment would indicate an adequate

138 population to sample from. In each site, we established four 1 ha plots with varying land use-

139 land cover types: agriculture, plantations (Areca nut and Banana), disturbed (logged) and

140 undisturbed forest (minimal use). To capture the variation in diet with respect to seasons, we

141 carried out the sampling in both dry (January to April) and wet (May to July) seasons, the latter

142 coinciding with the south-westerly monsoon.

143 Our protocol was approved by the Research Ethics Committee: Animal Care and Use,

144 Stellenbosch University (\#1260) and permission to capture anurans, was granted under the

145 permit of the Department of Environment and Forests, Andaman and Nicobar Islands

146 (\#CWLW/WL/134/350). Diet of anurans was determined using stomach flushing, a standard and

147 low-risk technique to determine prey consumed (Solé et al., 2005). Anurans were hand- 
148 captured between 1800 to $2200 \mathrm{hrs}$; stomach flushing was carried out within $3 \mathrm{~h}$ of capture.

149 We consciously avoided capture bias towards any particular size class, by actively searching for

150 anurans of all size classes. As our sampling focussed on sub-adult and adult $H$. tigerinus and was

151 completed in July (presumably before breeding and emergence of metamorphs) we did not

152 examine the diet of metamorphs. In order to avoid mortality, we did not stomach flush

153 individuals below $20 \mathrm{~mm} \mathrm{SVL}$ and hence, individuals of co-occurring Microhyla chakrapanii (ca.

154 10-30 mm SVL; Pillai, 1977) were not sampled After excluding native anurans which did not co-

155 occur with H. tigerinus, our samples included Duttaphrynus melanostictus (although its

156 taxonomic and geographic status is uncertain, Das 1999), Limnonectes and Fejervarya. We

157 conducted stomach flushing using a syringe ( $3 \mathrm{ml}$ to $10 \mathrm{ml}$ for anurans of $20 \mathrm{~mm}-50 \mathrm{~mm} \mathrm{SVL}$

158 and $60 \mathrm{ml}$ for anurans $>60 \mathrm{~mm} \mathrm{SVL}$ ), soft infusion tube, and water from site of capture. In

159 addition to SVL, we measured head width (HW) and lower jaw length (LL) of the anurans, using

160 a Vernier calliper ( $0.01 \mathrm{~mm}$ precision) and noted the sex. The stomach flushed individuals were

161 toe-clipped (following Grafe et al., 2011) to record the total number of recaptures ( $n=54)$.

162 Individuals were released back to the capture site post completion of the procedure.

163 We collected the expelled prey items in a transparent beaker and sieved the contents using a

164 mesh of $0.5 \mathrm{~mm}$. Prey items from each individual were classified up to a minimum of order

165 level, and further characterized by functional traits (hardness and motility, following

166 Vanhooydock et al., 2007). Length and width of intact prey were measured under an 8x

167 magnifying lens to the nearest $0.01 \mathrm{~mm}$ using a Vernier calliper and recorded along with the

168 prey's life stage (adult/larvae). We preserved all prey items in $70 \%$ ethanol. 
169 We also determined electivity of prey, based on prey consumption as compared to prey

170 availability. Terrestrial prey were measured using five pitfall traps in each 1 ha plot, which were

171 visited twice daily for a duration of three days (total of 30 trap occasions). Within each 1 ha

172 plot, the pitfalls were arranged in the four corners and one in the centre of the plot. We used

173 plastic traps, $80 \mathrm{~mm}$ in diameter and $300 \mathrm{~mm}$ high. A wet cloth was kept at the bottom to

174 provide refuge to trapped animals, so as to prevent any predation before sample collection. We

175 used chloroform soaked cotton balls to euthanize the invertebrate prey, prior to collection.

176 These prey items were also identified up to the order level and measured for length and width.

177 Our approach of estimating prey availability excludes flying evasive orders (e.g. adult

178 lepidopterans) and vertebrate prey.

179

180

181

182

183

184

185

186

187

188

\section{Data analyses}

We did not obtain adequate numbers of Duttaphrynus melanostictus $(n=4)$ individuals and hence they were not included in the analyses. We pooled samples from the three sites to examine diet at the species level for $\mathrm{H}$. tigerinus and genus level for Limnonectes and Fejervarya. We assessed the number, volume, and frequency (number of individuals with a given prey item in their stomach) of consumed prey under each taxonomic category. Volume was calculated using the formula of an ellipsoid, following Colli and Zamboni (1999),

volume $=\frac{4}{3} \pi\left(\frac{l}{2}\right)\left(\frac{w}{2}\right)^{2}$

where, I is prey length and $w$ is prey width. Prey items for which volume could not be calculated due to lack of measurement data (i.e. fragmented prey) were assigned the median prey volume 
189 for that order. We carried out a generalized linear model to test the relationship between body 190 size of anurans (SVL) and prey volume, after accounting for taxonomic identity of anurans. We 191 log transformed SVL to adhere to the assumption of normality and cube root transformed prey 192 volume, prior to the analysis.

193 In order to assess the overall importance of a prey category, based on the percentage of

194 number, frequency and volume, we used the Index of Relative Importance (IRI, Pinkas et al., 195 1971).

196 To test for diet overlap, we employed the MacArthur and Levins' index Ojk (MacArthur and 197 Levins 1967) in the pgirmess package (Giraudoux 2016); we built null models using the 198 'niche_null_model' function of the EcoSimR package (Gotelli et al., 2015) to test for statistical 199 significance of Ojk. We also assessed prey availability for each site across both dry and wet 200 seasons, using the Simpson's diversity index (Supplemental Information 1). We determined 201 electivity of terrestrial invertebrate prey by the anurans, using the Relativized Electivity Index 202 (Vanderploeg \& Scavia 1979). Following Measey (1998), we computed electivity for only those prey taxa with $\mathrm{n} \geq 10$ prey items for $H$. tigerinus and Limnonectes; given the low sample size for calculated only for agriculture and plantations; electivity for Fejervarya was considered only for one site with adequate sample size: Wandoor (Table 1). All analyses were carried out in the

207 statistical software R 3.4.1 (R Core Team 2017).

\section{RESULTS}


209 Overall, we sampled 798 individuals of the two native anurans and the invasive Hoplobatrachus

210 tigerinus (Table 1). We obtained 1478 prey items (H. tigerinus: 687, Limnonectes: 618,

211 Fejervarya: 173) belonging to 35 taxonomic categories in the stomach of 688 anurans (Table 2,

212 Supplemental Information 2). Vacuity index (i.e. proportion of empty stomachs) was higher in

213 the dry season (19.68\%) as compared to the wet season (8.67\%). Less than $4 \%$ of prey items

214 remained unidentified, mostly due to advanced levels of digestion. Hoplobatrachus tigerinus

215 consumed prey items under most of the taxonomic categories (29), followed by Limnonectes

216 (25), and Fejervarya (14). Vertebrates were consumed by both $H$. tigerinus and Limnonectes,

217 although the numeric and volumetric percentage of vertebrates consumed was higher for $H$.

218 tigerinus (2.62\%, 58.03\%) than Limnonectes (0.48\%, 5.16\%; Table 2). Based on IRI, coleopterans

219 and orthopterans constituted the major prey of $H$. tigerinus and Limnonectes, whereas,

220 formicids and coleopterans formed the majority in the diet of Fejervarya (Table 2).

221 The diet of $H$. tigerinus overlapped significantly with that of Limnonectes $(O j k=0.87$, lower-tail

$222 p>0.999$, upper-tail $p<0.001)$ but there was no significant overlap with Fejervarya $($ Ojk $=0.35$,

223 lower-tail $p=0.919$, upper-tail $p=0.08)$. The diet of the two native anurans overlapped

224 significantly $(O j k=0.58$, lower-tail $p=0.967$, upper-tail $p=0.03)$.

225 Based on availability of terrestrial invertebrates, prey electivity of all three anurans indicated a

226 positive relationship between predator-prey body sizes (Fig. 3). While the largest species, $H$.

227 tigerinus, strongly selected larger prey $\left(\geq 100 \mathrm{~mm}^{3}\right)$, the smallest anuran, Fejervarya, selected

228 for prey items smaller than $10 \mathrm{~mm}^{3}$; the medium sized Limnonectes chose small and medium-

229 sized prey items $\left(10 \mathrm{~mm}^{3}-500 \mathrm{~mm}^{3}\right)$, although the magnitude of electivity (positive or 
230

231

232

233

234

235

236

237

238

239

240

241

242

243

negative) was lowest for this species (Fig. 1; Fig. 3). We found a positive correlation between prey volume and body size of $H$. tigerinus $(\beta=1.93, \mathrm{SE}=0.21, p<0.001)$ and Limnonectes $(\beta=$ $0.88, \mathrm{SE}=0.25, p<0.001)$, but found no such relationship in case of Fejervarya $(\beta=-0.07, \mathrm{SE}=$ $0.33, p=0.83)$.The majority of prey consumed by the three anurans was hard, and evasive, although diet of Limnonectes included a relatively higher proportion of soft and sedentary prey (Table 3). Terrestrial prey were the dominant type in the diet of H. tigerinus $(91.29 \%)$, Limnonectes (93.18\%), and Fejervarya (99.34\%).

We observed several endemic vertebrate species in the diet of $H$. tigerinus, including the Andaman emerald gecko Phelsuma andamanensis $(n=1)$, Chakrapani's narrow mouthed frog Microhyla chakrapani (2), the Andaman skink Eutropis andamanensis (1), and Oates's blind snake Typhlophs oatesii (3). We also found Limnonectes (4), unidentified rodent (1), Lycodon sp. (1) and the invasive Calotes versicolor (3) in the diet of $H$. tigerinus (Supplemental Information 2). Limnonectes preyed upon a conspecific on one occasion and an unidentified anuran in another instance.

\section{DISCUSSION}

We expected the diet of invasive Hoplobatrachus tigerinus to overlap significantly with the diet of the two native anurans considered. However, we found a significant overlap only with Limnonectes, such that when prey is limited competition may arise. As expected, small vertebrates constituted a majority of $H$. tigerinus diet by volume, suggesting potential impact by predation on a large proportion of the endemic island fauna. Volume of prey elected was 
250 positively related to predator size (Fig. 3); within species, volume of prey consumed was

251 positively correlated with predator size for $\mathrm{H}$. tigerinus and Limnonectes only.

252 We observed $86 \%$ niche overlap between $H$. tigerinus and Limnonectes, which was statistically

253 significant in comparison to the constructed null model; whereas, niche overlap of $H$. tigerinus

254 with Fejervarya was not significant. On the other hand, prey electivity suggests that $H$. tigerinus

255 strongly elected for medium-sized and larger prey whereas small and medium-sized prey were

256 elected by Limnonectes (Fig. 3). This may result in competition for prey ranging from $10-500$

$257 \mathrm{~mm}^{3}$ between the two anurans, under the conditions of limited prey. Trophic competition in

258 amphibians may lead to a decrease in fitness (e.g. growth rate) and affect population level

259 processes (Benard \& Maher, 2011). Impact of invasive amphibians (post-metamorphic) via

260 trophic competition has been documented in fewer studies as compared to predation (Measey

261 et al., 2016), but this mechanism may affect taxa at various trophic levels (Smith et al., 2016).

262 Metamorphs of $\mathrm{H}$. tigerinus may also compete with both Fejervarya and Limnonectes as they

263 would fall under the same size class (20 mm-60mm; Daniels, 2005). The observed positive

264 correlation between body size and prey volume in the case of both $H$. tigerinus and

265 Limnonectes, also supports the notion that metamorphs of these species may compete for

266 small prey. Although our sampling did not evaluate the diet of $H$. tigerinus metamorphs, we

267 think this may be relevant as competition between juvenile Lithobates catesbeianus and small

268 native anurans has been previously documented on Daishan Island, China (Wu et al., 2005).

269 Evaluating dietary overlap is a pre-cursor to determining trophic competition due to invasive

270 populations, which do not have shared evolutionary history with native species. Dietary overlap 
271 in co-occurring species may be independently influenced by prey availability (Kuzmin, 1995),

272 prey taxa (Lima, 1998), prey size (Toft, 1981; Vignoli et al., 2009; Crnobrnja-Isailović, 2012) and

273 a combination of these factors. Therefore, it is essential to design studies and interpret dietary

274 patterns with reference to all three factors, in order to arrive at meaningful inferences on prey

275 consumed, dietary overlap, and probable subsequent competition (Kuzmin, 1990; but see

276 Kuzmin, 1995 regarding criteria for competition). Further, prey size should ideally be measured

277 in terms of volume, as it is known to be a better dietary descriptor (Vignoli \& Luiselli 2012).

278 Hoplobatrachus tigerinus preyed upon three classes of vertebrates (Amphibia, Reptilia, and

279 Mammalia), which accounted for a significant proportion of its diet by volume, although vertebrate prey was numerically inferior to invertebrates in the diet. Such major contribution to

281 the volume of prey by vertebrates (despite numerical inferiority) has been observed for

Lithobates catesbeianus and Xenopus laevis (Boelter et al., 2012; Vogt et al., 2017); anurophagy may also contribute significantly to the diet of many amphibians (Measey et al., 2015; Courant

et al., 2017). We observed several endemic species in the diet of $H$. tigerinus, which may become threatened if frequently preyed upon. Limnonectes was also consumed by $H$. tigerinus, thereby, indicating a potential two-pronged impact through predation and competition.

However, demographic change (if any) in Limnonectes, due to predation and competition by $\mathrm{H}$. tigerinus, was not evaluated in this study. The invasive $H$. tigerinus on the Andaman Islands reportedly consume poultry (Manish Chandi pers comm., Mohanty \& Measey, in review) and stream fish (NPM unpublished data), resulting in a potential economic impact. We expect the invasive $H$. tigerinus on Madagascar (Glaw and Vences, 2007) to similarly consume a large 
292 proportion of vertebrates in its diet and consider the invasion to be a threat to the highly

293 diverse small vertebrates of Madagascar.

294 Despite the presence of a large portion of vertebrates in the diet of $H$. tigerinus, its trophic

295 position (consistency of vertebrate prey consumption) can only be ascertained with stable

296 isotope analyses (Huckembeck et al., 2014). Although, diet analysis of invasive species can

297 identify vulnerable taxa and confirm at least 'minimal' to 'minor' levels of impact through

298 predation and competition (sensu Blackburn et al., 2014; Hawkins et al., 2015), such analysis

299 must be complimented with evidence of trophic level effects to evaluate the degree of impact

300 (Smith et al., 2016).

301 The large proportion of ants in the diet of Fejervarya does not necessarily prove specialization

302 for myrmecophagy. Hirai and Matsui (2000) inferred relatively weaker avoidance of ants by

303 Glandirana rugosa as compared to other anurans. Although we found the same pattern for

304 Fejervarya based on prey electivity $(E=-0.02)$, it does not prove weak avoidance either. As

305 social insects, ants may be disproportionately captured in the pitfall traps; therefore, it is

306 necessary to compliment diet studies on potentially myrmephagous predators with additional

307 evidence (e. g. cafeteria experiments). Hoplobatrachus tigerinus and Fejervarya chose evasive

308 prey, suggesting that these two species are mostly ambush ('sit and wait') predators;

309 Limnonectes elected sedentary prey along with other prey types, indicating a combination of

310 'active search' and 'sit and wait' foraging (Table 3; Huey \& Pianka, 1981; Vanhooydonck et al.,

311 2007). Generally, soft bodied prey are considered to provide more nutrition by size as

312 compared to hard prey and therefore, it is hypothesized that species will select soft prey more 
313 often than hard prey, which in turn is dependent on prey availability by season (Measey et al.,

314 2011; Carne \& Measey 2013). However, we find that diet does not appear to vary considerably

315 across the seasons and is governed more by size than hardness of prey (Fig. 3; Werner et al., 316 1995).

317 Although our sampling for diet analysis by stomach flushing was adequate (Table 1), our

318 assessment of prey availability did not include flying invertebrates and vertebrates, which

319 prevents us from carrying out electivity analyses on these taxa.

CONCLUSION

Diet analyses of Hoplobatrachus tigerinus confirmed our first hypothesis, i.e. significant predation of $H$. tigerinus on endemic vertebrates (hypothesis 1 ) and partially supported the second hypothesis of a high diet overlap with native anurans (hypothesis 2 ) indicating potential competition; overlap was significant only for the large-bodied Limnonectes. Given the observed high density of $H$. tigerinus in human modified habitats on the Andaman archipelago (NPM unpublished data), trophic competition and predation by $H$. tigerinus may have a significant impact on native anuran populations in these habitats. Pursuing our additional aim of characterizing anuran foraging modes, we determined the foraging strategy of $H$. tigerinus and Fejervarya as ambush foraging ('sit and wait') and that of Limnonectes to be a combination of 'active search' and 'sit and wait' foraging. In addition to quantifying the trophic niche of anurans belonging to three genera, we stress the necessity to evaluate prey availability and

332 volume in future studies for meaningful insights into diet of amphibians. 
334 We would like to thank the Department of Environment and Forests, Andaman and Nicobar

335 Islands for granting permitsthe Andaman \& Nicobar Environment Team (ANET) for facilitating

336 field work, Saw Issac and Saw Sathaw for collecting part of the data and help during field work.

337 NPM would like to acknowledge the support and advice of Dr. Karthikeyan Vasudevan, Dr.

338 Harikrishnan S., Dr. Manish Chandi, and Ashwini Mohan during the study.

\section{REFERENCES}

340 Andrews HV, Sankaran V. 2002. Sustainable management of protected areas in the Andaman

341 and Nicobar Islands. Port Blair: Andaman and Nicobar Environment Team.

342 Beard KH, Pitt WC. 2005. Potential consequences of the coqui frog invasion in Hawaii. Diversity

343 and Distributions, 11:427-433.

344 Blackburn TM, EssI F, Evans T, Hulme PE, Jeschke JM, Kühn I, Kumschick S, Marková Z, Mrugała

345 A, Nentwig W, Pergl J. 2014. A unified classification of alien species based on the magnitude of

346 their environmental impacts. PLoS biology, 12(5), p.e1001850.

347 Boelter RA, Kaefer IL, Both C, Cechin S. 2012. Invasive bullfrogs as predators in a Neotropical

348 assemblage: What frog species do they eat? Animal Biology, 62(4):397-408.

349 Boland CRJ. 2004. Introduced cane toads Bufo marinus are active nest predators and

350 competitors of rainbow bee-eaters Meropsornatus: observational and experimental evidence.

351 Biological conservation, 120(1):53-62.

352 Carne L and Measey J. 2013. Chameleons on the cruise: seasonal differences in prey

353 choice of two dwarf chameleons. Herpetological Journal. 23:221-227. 
354 Chandramouli SR, Khan T, Yathiraj R, Deshpande N, Yadav S, Tejpal C, de Groot S, Lammes I.

355 2015. Diversity of amphibians in Wandoor, South Andaman, Andaman and Nicobar Islands, 356 India. Alytes, 32.

357 Chandramouli SR. 2016. Rediscovery and redescription of a little known, insular endemic frog, 358 Ingerana charlesdarwini (Das, 1998)(Amphibia: Anura: Dicroglossidae) from the Andaman 359 Islands, Bay of Bengal. Alytes, 33.

RK. 2016. A new genus and species of arboreal toad with phytotelmonous larvae, from the Andaman Islands, India (Lissamphibia, Anura, Bufonidae). ZooKeys, (555), p.57. Natural History. Tropical Natural History, 18(1):40-53.

Choi RT, Beard KH. 2012. Coqui frog invasions change invertebrate communities in Hawaii.

Biological Invasions, 14(5):939-948.

Colli GR, Zamboni DS. 1999. Ecology of the worm-lizard Amphisbaena alba in the native populations?. PeerJ, 5, p.e3250. 
373 Crossland MR, Brown GP, Anstis M, Shilton CM, and Shine R. 2008. Mass mortality of native

374 anuran tadpoles in tropical Australia due to the invasive cane toad (Bufo marinus). Biological

375 Conservation, 141(9): 2387-2394.

376 D’Amore A, Kirby E, Hemingway V. 2009. Reproductive interference by an invasive species: an

377 evolutionary trap. Herpetological Conservation and Biology, 4(3):325-330.Daniels RR. 2005.

378 Amphibians of peninsular India. - Universities Press, India.

379 Das I. 1999. Biogeography of the amphibians and reptiles of the Andaman and Nicobar Islands.

380 Tropical Island Herpetofauna-Origin, Current Diversity and Conservation, 43-77.

381 de Oliveira EG, Haddad CF. 2015. Diet Seasonality and Feeding Preferences of Brachycephalus

382 pitanga (Anura: Brachycephalidae). Journal of Herpetology, 49(2):252-256.

Datta AK, Khaledin S. 2017.Observations on an Indian bullfrog swallowing an Asian common

toad, and a Checkered keelback on a skipper frog. Zoo's Print,32:28-29

Dutta SK. 1997. Amphibians of India and Sri Lanka: checklist and bibliography. Odyssey

Publishing House.

Forest Statistics 2013, accessed from http://forest.and.nic.in/ on 15 ${ }^{\text {th }}$ Aug 2017.

Gardiner JS. 1906. Notes on the distribution of the land and marine animals, with a list of the land plants and some remarks on the coral reefs. - In Gardiner, J.S. (ed.), The fauna and geography of the Maldive and Laccadive archipelagos. Cambridge University Press, Cambridge. pp. 1046-1057. 
392 Garner TWJ, Perkins MW, Govindarajulu P, Seglie D, Walker S, Cunningham AA, Fisher MC.

393 2006. The emerging amphibian pathogen Batrachochytrium dendrobatidis globally infects

394 introduced populations of the North American bullfrog, Rana catesbeiana. Biology letters 2:455-

395459.

396 Giraudoux P. 2016. pgirmess: data analysis in ecology. R package version 1.6.4. Available at

397 http://CRAN.R-project.org/ package=pgirmess.

Glaw F, Vences M. 2007. A field guide to the amphibians and reptiles of Madagascar. ISBN-13:

399

9783929449037.

Gotelli NJ, Hart EM, Ellison AM. 2015. EcoSimR: Null model analysis for ecological data. R package version 0.1.0. Available at http:// github.com/ gotellilab/EcoSimR. viable method for marking anurans. Journal of Herpetology, 45(1):28-35.

Greenlees MJ, Brown GP, Webb JK, Phillips BL, Shine R. 2006. Effects of an invasive anuran [the 405 cane toad (Bufo marinus)] on the invertebrate fauna of a tropical Australian floodplain. Animal 406 Conservation, 9(4):431-438.

Greenlees, MJ, Brown, GP, Webb JK, Phillips BL, Shine R. 2007. Do invasive cane toads (Chaunus marinus) compete with Australian frogs (Cyclorana australis)?. Austral Ecology, 32(8):900-907. studies from Andaman and Nicobar islands, with an updated checklist. In: Ramakrishna, 
411 Raghunathan C, Sivaperuman C (eds) Recent trends in biodiversity of Andaman and Nicobar

412 Islands. Zoological Survey of India, Kolkata, pp 387-398.

413 Hawkins CL, Bacher S, EssI F, Hulme PE, Jeschke JM, Kühn I, Kumschick S, Nentwig W, Pergl J,

414 Pyšek P, Rabitsch W. 2015. Framework and guidelines for implementing the proposed IUCN

415 Environmental Impact Classification for Alien Taxa (EICAT). Diversity and Distributions. 21:1360-

4161363.

417 Hirschfeld M, Rödel MO. 2011. The diet of the African Tiger Frog, Hoplobatrachus occipitalis, in 418 northern Benin. Salamandra, 47(3):125-132.

419 Hodgkison S, Hero JM. 2003. Seasonal, sexual and ontogenetic variations in the diet of the

420 'declining' frogs Litoria nannotis, Litoria rheocola and Nyctimystes dayi. Wildlife

421 Research, 30(4):345-354.

422 Huey RB and Pianka ER. 1981. Ecological Consequences of Foraging Mode. Ecology, 62:991-999.

423 Kats LB, Ferrer RP. 2003. Alien predators and amphibian declines: review of two decades of

424 science and the transition to conservation. Diversity and Distributions, 9:99-110.

425 Khatiwada JR, Ghimire S, Khatiwada SP, Paudel B, Bischof R, Jiang J, Haugaasen T. 2016. Frogs as

426 potential biological control agents in the rice fields of Chitwan, Nepal. Agriculture, Ecosystems

427 and Environment, 230:307-314.

428 Kraus F. 2015. Impacts from Invasive Reptiles and Amphibians. Annual Review of Ecology,

429 Evolution, and Systematics, 46:75-97. 
430 Lafferty KD, Page CJ 1997. Predation on the endangered tidewater goby, Eucyclogobius

431 newberryi, by the introduced African clawed frog, Xenopus laevis, with notes on the frog's

432 parasites. Copeia, 1997(3):589-592.

433 Liu X, Rohr JR and Li Y. 2013. Climate, vegetation, introduced hosts and trade shape a global

434 wildlife pandemic. Proc. R. Soc. B, 280(1753):20122506.

435 Liu X, Luo Y, Chen J, Guo Y, Bai C and Li Y. 2015. Diet and prey selection of the Invasive American

436 bullfrog (Lithobates catesbeianus) in southwestern China. Asian herpetological research, 6:34-

43744.

438 MacArthur R, Levins R (1967). The limiting similarity, convergence, and divergence of coexisting 439 species. American naturalist, 377-385.

440 Measey, GJ. 1998. Diet of feral Xenopuslaevis (Daudin) in South Wales, UK. Journal of Zoology, $441 \quad 246(3): 287-298$.

442 Measey GJ, Rebelo AD, Herrel A, Vanhooydonck B, Tolley KA. 2011. Diet, morphology and 443 performance in two chameleon morphs: do harder bites equate with harder prey? Journal of 444 Zoology, 285:247-255.

445 Measey GJ, Vimercati G, De Villiers FA, Mokhatla MM, Davies SJ, Edwards S, Altwegg R. 446 2015.2015. Frog eat frog: exploring variables influencing anurophagy. PeerJ, 3, e1204.

447 Measey GJ, Vimercati G, De Villiers FA, Mokhatla MM, Davies SJ, Thorp CJ, Rebelo AD, 448 Kumschick S. 2016. A global assessment of alien amphibian impacts in a formal

449 framework. Diversity and Distributions, 22:970-981. 
450 Mohanty NP, Measey J. in review. Reconstructing biological invasions using public surveys: a

451 new approach to retrospectively assess spatio-temporal changes in invasive spread. Biological

452 Invasions.

453 Myers N, Mittermeier RA, Mittermeier CG, Da Fonseca GA, Kent J. 2000. Biodiversity hotspots 454 for conservation priorities. Nature, 403(6772): p.853.

455 Nunez MA, Pauchard A. 2010. Biological invasions in developing and developed countries: does 456 one model fit all? Biological invasions, 12:707-714.

457 Oliveira BF, São-Pedro VA, Santos-Barrera G, Penone C and Costa GC. 2017. AmphiBIO, a global 458 database for amphibian ecological traits. Scientific data, 4:170123.

459 Padhye A, Manamendra-Arachchi K, de Silva A, Dutta S, Kumar Shrestha T, Bordoloi S, 460 Papenfuss T, Anderson S, Kuzmin S, Khan MS, Nussbaum R. 2008. Hoplobatrachus tigerinus. The 461 IUCN Red List of Threatened Species. e.T58301A11760496.

462 Pinkas L, Oliphant MS, Iverson ZL. 1971. Food habits of albacore bluefin, tuna and bonito in 463 California waters. California Department of Fish and Game Bulletin, 152:1-350.Pitt W, Vice D, 464 Pitzler M. 2005. Challenges of invasive reptiles and amphibians. Proceedings of Wildlife Damage 465 Management Conference.

466 Pyšek P, Richardson DM, Pergl J, Jarošík V, Sixtova Z, Weber E. 2008. Geographical and 467 taxonomic biases in invasion ecology. Trends in Ecology \& Evolution, 23:237-244.

468 R Core Team. 2017. R: a language and environment for statistical computing. Vienna: R 
469 Foundation for Statistical Computing. Available at https://www.R-project.org/.

470 Seebens H, Blackburn TM, Dyer EE, Genovesi P, Hulme PE, Jeschke JM, Pagad S, Pyšek P, Winter

471 M, Arianoutsou M, Bacher S. 2017. No saturation in the accumulation of alien species

472 worldwide. Nature communications, 8: p.14435.

473 Shannon CE, Weaver W. 1964. The mathematical theory of communication. Urbana: University

474 of Illinois Press.

475 Shine R. (2010). The ecological impact of invasive cane toads (Bufo marinus) in Australia. The

476 Quarterly Review of Biology, 85(3):253-291.

477 Simberloff D, Martin JL, Genovesi P, Maris V, Wardle DA, Aronson J, Courchamp F, Galil B,

478 García-Berthou E, Pascal M, Pyšek, P. 2013. Impacts of biological invasions: what's what and the

479 way forward. Trends in Ecology \& Evolution, 28:58-66.

480 Solé M, Beckmann O, Pelz B, Kwet A, Engels W. 2005. Stomach-flushing for diet analysis in

481 anurans: an improved protocol evaluated in a case study in Araucaria forests, southern Brazil.

482 Studies on Neotropical Fauna and Environment, 40:23-28.

483 Vanderploeg HA, Scavia D. 1979. Calculation and use of selectivity coefficients of feeding:

484 zooplankton grazing. Ecological Modelling, 7:135-149.

485 Vanhooydonck B, Herrel A, Van Damme R. 2007. Interactions between habitat use, behavior,

486 and the trophic niche of lacertid lizards. In: Lizard Ecology: The Evolutionary Consequences of

487 Foraging Mode, ed. S. M. Reilly, L. D. McBrayer and D. B. Miles. Cambridge University Press. 
488 Vogt S, De Villiers FA, Ihlow F, Rödder D, Measey GJ. 2017. Competition and feeding ecology

489 in two sympatric Xenopus species (Anura: Pipidae). PeerJ, 5, p.e3130.Werner EE, Wellborn GA,

490 McPeek MA. 1995. Diet composition in postmetamorphic bullfrogs and green frogs:

491 implications for interspecific predation and competition. Journal of Herpetology, 600-607.

492 Wu Z, Li Y, Wang Y, Adams MJ. 2005. Diet of introduced bullfrogs (Rana catesbeiana): predation

493 on and diet overlap with native frogs on Daishan Island, China. Journal of Herpetology,

$49439(4): 668-674$. 
Figure 1 (on next page)

Snout-vent length of three species of anurans used for dietassessment.

Individuals belong to the invasive Indian bullfrog Hoplobatrachus tigerinus and the native Limnonectes spp. and Fejervarya spp., sampled at three locations on the Andaman archipelago. 


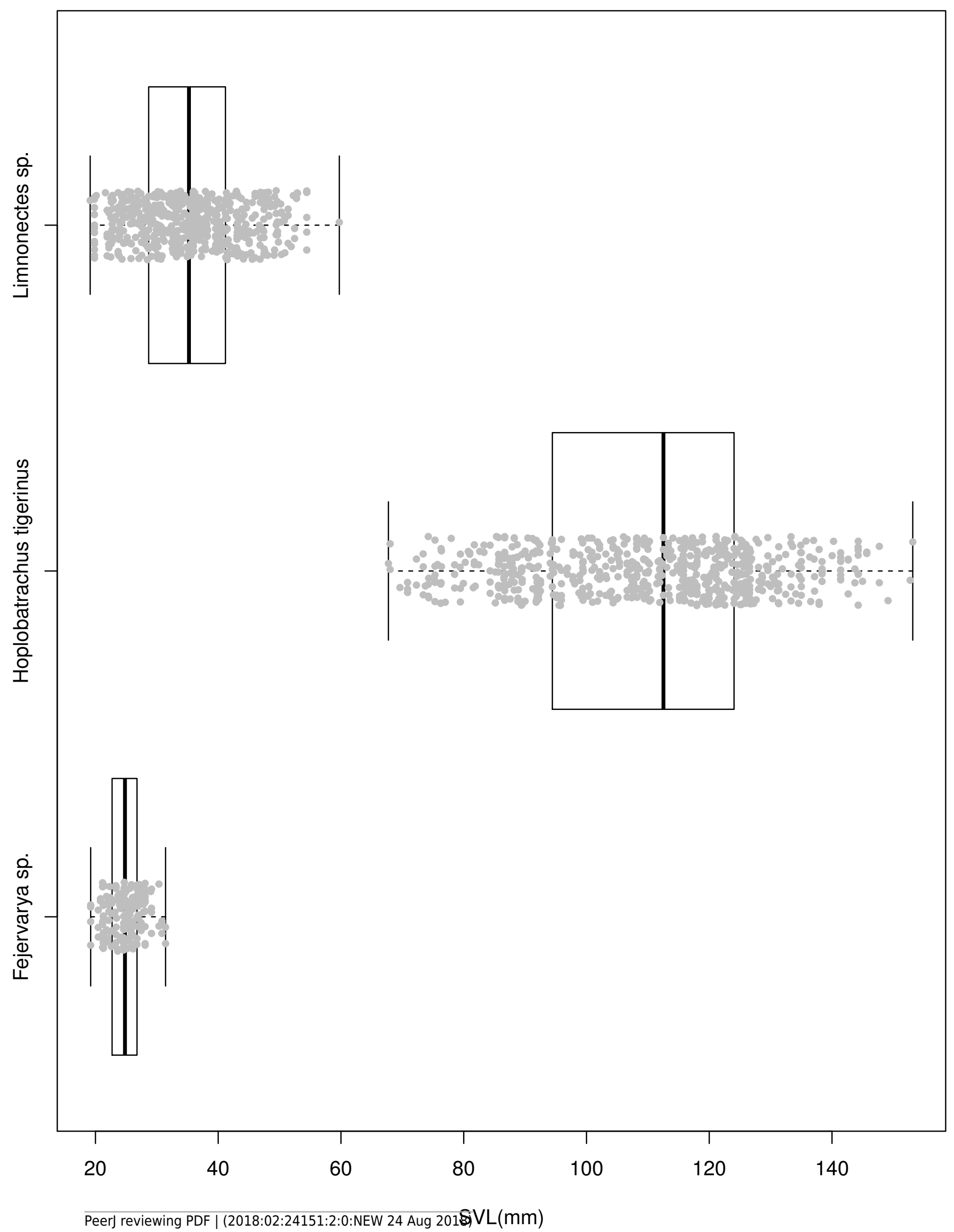




\section{Figure 2}

Study area map showing the major islands of theAndaman archipelago and the three sampling locations.

Diet assessment of Hoplobatrachus tigerinus, Limnonectes spp., and Fejervarya spp. were carried out from February 2017 - July 2017. 

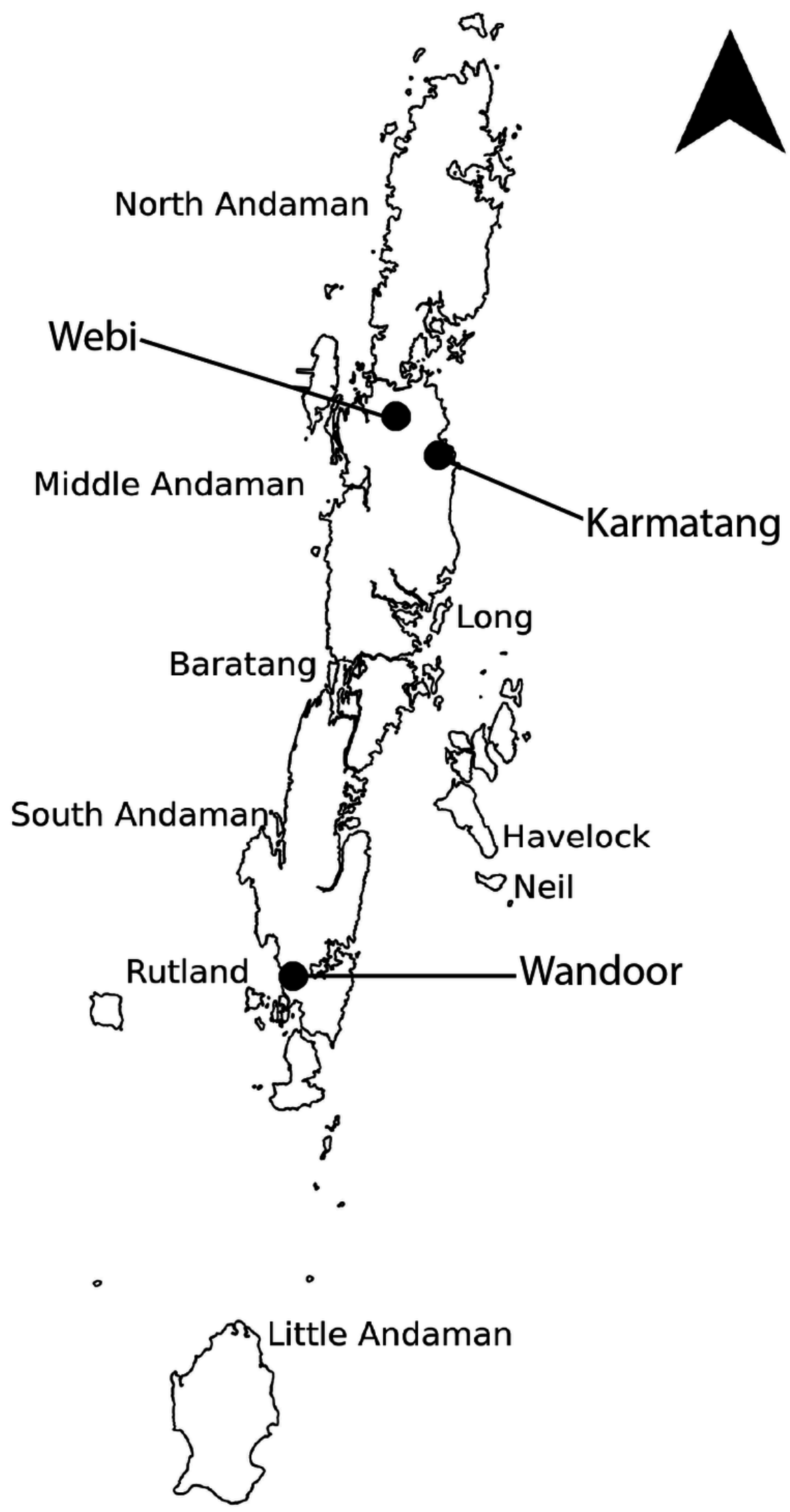

$\begin{array}{lllll}0 & 25 & 50 & 75 & 100 \mathrm{~km}\end{array}$ 
Figure 3

Prey electivity in terms of volume, by the invasive Hoplobatrachustigerinus and native Limnonectes spp.and Fejervarya spp.

Prey electivity based on prey consumption and availability, at three sites on the Andaman archipelago.

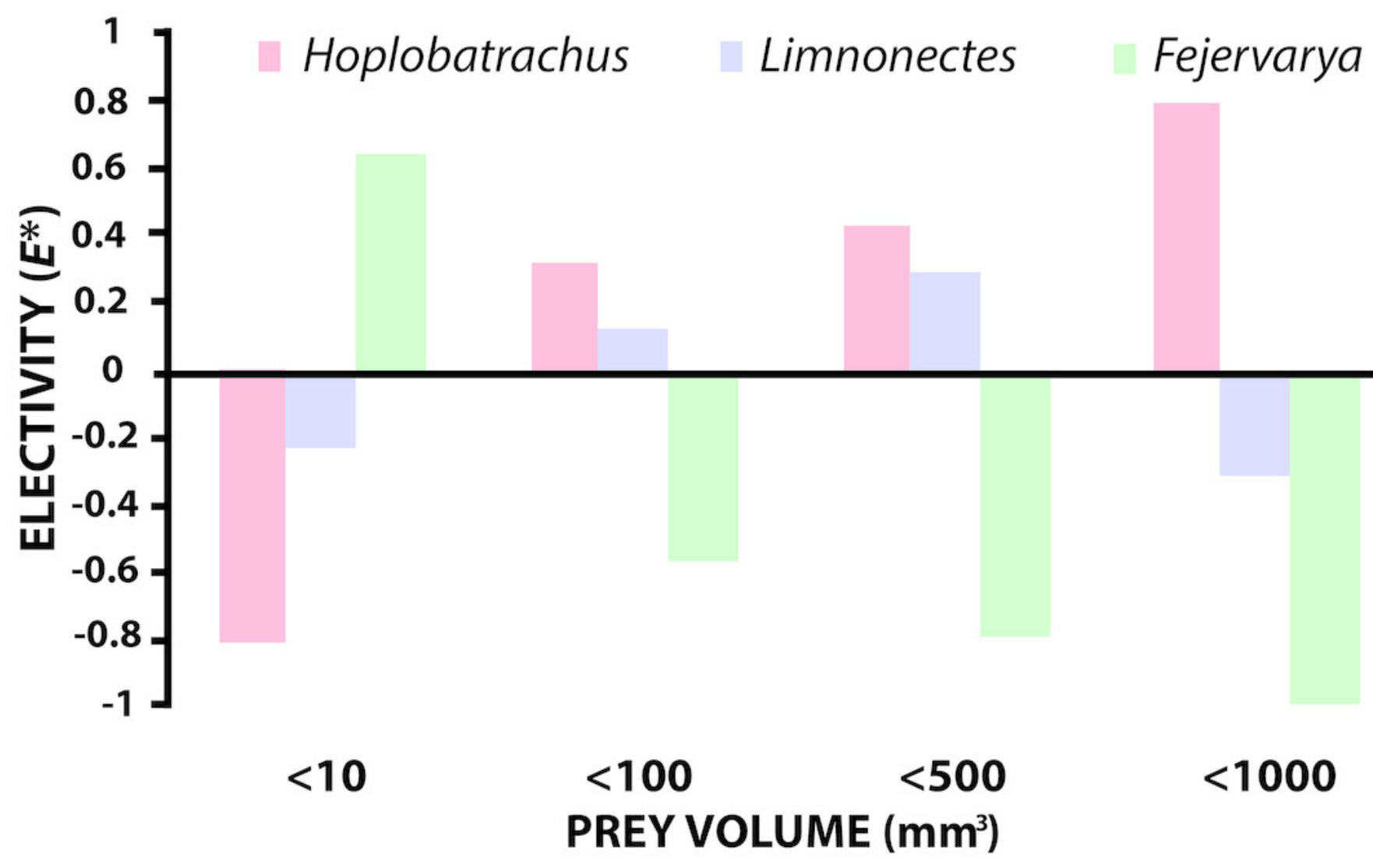




\section{Table $\mathbf{1}$ (on next page)}

Sampling effort for diet assessment of theinvasive Hoplobatrachus tigerinus andnative Limnonectes spp. and Fejervarya spp.

Sampling carried out in four habitat types across two seasons, at three sampling locations on the Andaman Islands. 
1 Table 1: Sampling effort for diet assessment of the invasive Hoplobatrachus tigerinus and native

2 Limnonectes spp. and Fejervarya spp. Sampling carried out in four habitat types across two

3 seasons, at three sampling locations on the Andaman Islands.

4

\begin{tabular}{|c|c|c|c|c|c|c|c|c|}
\hline & \multicolumn{2}{|c|}{ Agriculture } & \multicolumn{2}{|c|}{ Plantation } & \multicolumn{2}{|c|}{ Disturbed Forest } & \multicolumn{2}{|c|}{ Undisturbed Forest } \\
\hline & dry & wet & dry & wet & dry & wet & dry & wet \\
\hline \multicolumn{9}{|l|}{ H. tigerinus } \\
\hline Karmatang & 41 & 35 & 29 & 29 & 0 & 0 & 0 & 0 \\
\hline Webi & 32 & 35 & 48 & 38 & 0 & 0 & 0 & 0 \\
\hline Wandoor & 0 & 0 & 38 & 33 & 0 & 0 & 0 & 0 \\
\hline \multicolumn{9}{|l|}{ Limnonectes } \\
\hline Karmatang & 0 & 17 & 5 & 26 & 0 & 25 & 0 & 22 \\
\hline Webi & 14 & 17 & 19 & 26 & 13 & 17 & 13 & 17 \\
\hline Wandoor & 7 & 21 & 17 & 29 & 19 & 11 & 30 & 10 \\
\hline \multicolumn{9}{|l|}{ Fejervarya } \\
\hline Karmatang & 0 & 0 & 0 & 0 & 0 & 0 & 0 & 0 \\
\hline Webi & 1 & 0 & 0 & 0 & 1 & 0 & 0 & 0 \\
\hline Wandoor & 19 & 17 & 13 & 2 & 10 & 0 & 2 & 0 \\
\hline
\end{tabular}

5 


\section{Table 2 (on next page)}

Diet of Hoplobatrachus tigerinus $(n=687)$, Limnonectes $(n=618)$ and Fejervarya $(n=$ 173) in three sites on the Andaman archipelago

Diet described in terms of percentage $\mathrm{N}$ - prey abundance, $\mathrm{V}$ - volume, $\mathrm{F}$ - frequency of occurrence in anurans, and IRI - Index of relative importance. 
1 Table 2: Diet of Hoplobatrachus tigerinus $(n=687)$, Limnonectes $(n=618)$ and Fejervarya $(n=173)$, described in terms of

2 percentage $\mathrm{N}$ - prey abundance, $\mathrm{V}$ - volume, $\mathrm{F}$ - frequency of occurrence in anurans, and IRI - Index of relative importance.

\begin{tabular}{|c|c|c|c|c|c|c|c|c|c|c|c|c|}
\hline \multicolumn{5}{|c|}{ Hoplobatrachus tigerinus $(n=687)$} & \multicolumn{4}{|c|}{ Limnonectes $(n=618)$} & \multicolumn{4}{|c|}{ Fejervarya $(n=173)$} \\
\hline Prey & $\mathrm{N} \%$ & V\% & $\mathrm{F} \%$ & $\mid \mathrm{RI}$ & $\mathrm{N} \%$ & V\% & $\mathrm{F} \%$ & IRI & $\mathrm{N} \%$ & V\% & $\mathrm{F} \%$ & $\mid \mathrm{RI}$ \\
\hline Acari & 0 & 0 & 0 & 0 & 0.32 & 0.006 & 0.39 & 0.12 & 0.57 & 0.14 & 0.84 & 0.61 \\
\hline Agamidae & 0.43 & 50.44 & 0.57 & 29.07 & 0 & 0 & 0 & 0 & 0 & 0 & 0 & 0 \\
\hline Amphipoda & 0 & 0 & 0 & 0 & 0.48 & 0.07 & 0.58 & 0.32 & 0 & 0 & 0 & 0 \\
\hline Anura & 0.87 & 4.95 & 1.14 & 6.65 & 0.32 & 5.12 & 0.39 & 2.12 & 0 & 0 & 0 & 0 \\
\hline Aranae & 3.20 & 0.73 & 4 & 15.74 & 7.60 & 2.27 & 8.59 & 84.93 & 7.51 & 7.75 & 10.16 & 155.23 \\
\hline Arthropoda & 6.55 & 0 & 8.57 & 56.22 & 5.50 & 0 & 6.64 & 36.53 & 0.57 & 0 & 0.84 & 0.48 \\
\hline Blattaria & 1.45 & 0.33 & 1.90 & 3.42 & 1.29 & 0.71 & 1.56 & 3.14 & 0 & 0 & 0 & 0 \\
\hline Chilopoda & 3.35 & 6.15 & 2.85 & 27.15 & 3.23 & 2.75 & 3.9 & 23.41 & 1.15 & 7.62 & 1.69 & 14.88 \\
\hline Coleoptera & 29.73 & 12.14 & 24.57 & 1029.14 & 15.85 & 10.34 & 15.42 & 404.29 & 9.24 & 20.50 & 12.71 & 378.16 \\
\hline Brachyura & 0.58 & 2.40 & 0.76 & 2.27 & 0.16 & 0.81 & 0.19 & 0.19 & 0 & 0 & 0 & 0 \\
\hline
\end{tabular}




\begin{tabular}{|c|c|c|c|c|c|c|c|c|c|c|c|c|}
\hline Dermaptera & 0.14 & 0.009 & 0.19 & 0.02 & 1.61 & 0.20 & 1.95 & 3.55 & 0 & 0 & 0 & 0 \\
\hline Diplopoda & 0.87 & 0.07 & 0.76 & 0.72 & 3.55 & 0.73 & 3.12 & 13.41 & 0 & 0 & 0 & 0 \\
\hline Diptera & 1.89 & 0.56 & 1.52 & 3.74 & 4.04 & 0.09 & 3.9 & 16.15 & 14.45 & 3.38 & 14.40 & 256.95 \\
\hline Formicidae & 3.93 & 0.37 & 3.80 & 16.42 & 10.19 & 0.24 & 8.00 & 83.58 & 38.72 & 5.80 & 23.72 & 1056.60 \\
\hline Gastropoda & 4.22 & 0.71 & 4 & 19.76 & 3.23 & 1.5 & 3.32 & 15.72 & 0 & 0 & 0 & 0 \\
\hline Geckonnidae & 0.14 & 0.45 & 0.19 & 0.11 & 0 & 0 & 0 & 0 & 0 & 0 & 0 & 0 \\
\hline Hemiptera & 0.58 & 0.19 & 0.76 & 0.59 & 2.10 & 0.35 & 2.34 & 5.77 & 5.20 & 10.96 & 5.08 & 82.18 \\
\hline Hymenoptera & 0.14 & 0.004 & 0.19 & 0.02 & 0 & 0 & 0 & 0 & 1.15 & 0.86 & 0.84 & 1.70 \\
\hline Insecta & 1.45 & 0 & 1.90 & 2.77 & 1.29 & 0 & 1.36 & 1.76 & 6.35 & 0 & 9.32 & 59.27 \\
\hline Isoptera & 2.62 & 0.24 & 2.09 & 6.01 & 7.44 & 1.88 & 4.49 & 41.89 & 2.31 & 0.87 & 3.38 & 10.81 \\
\hline Lacertidae & 0.29 & 0.90 & 0.38 & 0.45 & 0 & 0 & 0 & 0 & 0 & 0 & 0 & 0 \\
\hline Lepidoptera & 1.31 & 0.24 & 1.33 & 2.07 & 0.48 & 0.14 & 0.39 & 0.24 & 0 & 0 & 0 & 0 \\
\hline Leplarva & 6.26 & 3.01 & 7.42 & 68.95 & 6.63 & 5.95 & 6.64 & 83.59 & 3.46 & 15.08 & 4.23 & 78.61 \\
\hline Mantodea & 0.29 & 0.72 & 0.38 & 0.38 & 0 & 0 & 0 & 0 & 0 & 0 & 0 & 0 \\
\hline Odonata & 0.72 & 0.07 & 0.95 & 0.76 & 0.16 & 0.04 & 0.19 & 0.04 & 0 & 0 & 0 & 0 \\
\hline
\end{tabular}




\begin{tabular}{|c|c|c|c|c|c|c|c|c|c|c|c|c|}
\hline Oligochaeta & 1.31 & 0.77 & 1.52 & 3.18 & 4.69 & 54.54 & 4.10 & 242.95 & 0 & 0 & 0 & 0 \\
\hline Opilionida & 0 & 0 & 0 & 0 & 0 & 0 & 0 & 0 & 0 & 0 & 0 & 0 \\
\hline Orthoptera & 24.48 & 12.62 & 24.19 & 897.74 & 13.26 & 9.45 & 14.84 & 337.34 & 3.46 & 20.01 & 5.08 & 119.39 \\
\hline Rodentia & 0.14 & 0 & 0.19 & 0.02 & 0 & 0 & 0 & 0 & 0 & 0 & 0 & 0 \\
\hline Scincidae & 0.14 & 0.62 & 0.19 & 0.14 & 0 & 0 & 0 & 0 & 0 & 0 & 0 & 0 \\
\hline Serpentes & 0.58 & 0.67 & 0.76 & 0.95 & 0.16 & 0.04 & 0.19 & 0.04 & 0 & 0 & 0 & 0 \\
\hline Siphonaptera & 0 & 0 & 0 & 0 & 0 & 0 & 0 & 0 & 0.57 & 0.075 & 0.84 & 0.55 \\
\hline Gastropoda & 0.29 & 0.27 & 0.38 & 0.21 & 0.80 & 1.97 & 0.78 & 2.17 & 0 & 0 & 0 & 0 \\
\hline Unidentified & 1.89 & 0.26 & 2.47 & 5.35 & 5.33 & 0.69 & 6.44 & 38.87 & 5.20 & 6.92 & 6.77 & 82.19 \\
\hline Zygentoma & 0 & 0 & 0 & 0 & 0.16 & 0.01 & 0.19 & 0.03 & 0 & 0 & 0 & 0 \\
\hline
\end{tabular}

4 


\section{Table 3(on next page)}

Prey electivity ( $\left.E^{\prime}\right)$ of the invasive Hoplobatrachus tigerinus and native Limnonectes and Fejervarya based on prey hardness and motility, in three sites of the Andaman archipelago

Classification of prey hardness and motility following Vanhooydonck et al. (2007). Sampling carried out in four habitat types across two seasons, at three sampling locations on the Andaman Islands. 
1 Table 3. Prey electivity ( $\left.E^{\prime}\right)$ of the invasive Hoplobatrachus tigerinus and native Limnonectes and

2 Fejervarya based on prey hardness and motility, following Vanhooydonck et al. (2007).

3 Sampling carried out in four habitat types across two seasons, at three sampling locations on

4 the Andaman Islands.

5

H. tigerinus

\begin{tabular}{lrr} 
& dry & \multicolumn{2}{c}{ wet } \\
soft & -0.10 & -0.31 \\
medium & 0.80 & -0.07 \\
hard & -0.59 & 0.32
\end{tabular}

Limnonectes

\begin{tabular}{lrr} 
& dry & \multicolumn{2}{c}{ wet } \\
soft & 0.52 & 0.14 \\
medium & 0.15 & -0.09 \\
hard & -0.52 & -0.09
\end{tabular}

Fejervarya

\begin{tabular}{lrr} 
& dry & \multicolumn{2}{c}{ wet } \\
soft & 0.14 & -0.18 \\
medium & -0.45 & -0.43 \\
hard & -0.01 & 0.38 \\
\hline
\end{tabular}

\begin{tabular}{|c|c|c|}
\hline \multicolumn{2}{|c|}{ dry } & wet \\
\hline sedentary & -0.12 & -0.22 \\
\hline medium & -0.70 & 0.20 \\
\hline evasive & 0.85 & -0.01 \\
\hline
\end{tabular}

\begin{tabular}{|c|c|c|}
\hline & & et \\
\hline sedentary & 0.41 & 0.15 \\
\hline med & -0.46 & -0.11 \\
\hline I & 0.31 & -0.06 \\
\hline
\end{tabular}

\begin{tabular}{lrr} 
& dry & \multicolumn{2}{c}{ wet } \\
sedentary & 0.01 & -0.33 \\
medium & 0.10 & 0.49 \\
evasive & -0.34 & -0.45 \\
\hline
\end{tabular}

6 